\title{
Diagnostic Challenges of Acute Abdominal Emergencies in Spinal Cord Injury Patients: A Clinical Perspective
}

\author{
Allan Stolarski ${ }^{1,2}$, Katherine $\mathrm{He}^{1,3}$, Edward Whang ${ }^{1,3}$, and Gentian Kristo ${ }^{1,3, *}$ \\ ${ }^{1}$ Department of Surgery, Veterans Affairs Boston Healthcare System, Boston, MA, USA \\ ${ }^{2}$ Department of Surgery, Boston Medical Center, Boston University, Boston, MA, USA \\ ${ }^{3}$ Department of Surgery, Brigham and Women's Hospital, Harvard Medical School, Boston, MA, USA
}

*Corresponding author: Department of Surgery, Veterans Affairs Boston Healthcare System (112-C), 1400 VFW Parkway, West Roxbury, Boston, MA 02132, USA, Tel: +1-857-2013-6966; Fax: +1-857-203-5738; E-mail: gentian.kristo@va.gov

Received: 22 Apr, 2020 | Accepted: 24 Apr, 2020 | Published: 29 Apr, 2020

Citation: Stolarski A, He K, Whang E, Kristo G (2020) Diagnostic Challenges of Acute Abdominal Emergencies in Spinal Cord Injury Patients: A Clinical Perspective. J Surg Open Access 6(4): dx.doi.org/10.16966/2470-0991.215

Copyright: (c) 2020 Stolarski A, et al. This is an open-access article distributed under the terms of the Creative Commons Attribution License, which permits unrestricted use, distribution, and reproduction in any medium, provided the original author and source are credited.

\begin{abstract}
Background: Early diagnosis of acute abdominal emergencies in Spinal Cord Injury (SCI) patients is the most important factor for improving outcomes. However, the diagnosis and management of acute abdomen in $\mathrm{SCl}$ patients remains a significant challenge due to alterations in their abdominal somatic and visceral sensation, as well as coexisting chronic abdominal conditions prevalent in this population. The purpose of this paper is to provide a contemporary review of the diagnosis and management of abdominal emergencies in spinal cord injury patients.
\end{abstract}

Data Sources: Peer-reviewed, English language studies published between 1985 and 2019 reporting on acute abdomen in SCl patients. Reference lists of articles were reviewed to identify additional studies.

Conclusions: In addition to understanding the neurological consequences of spinal cord injury and maintaining a high clinical suspicion for abdominal emergencies in $\mathrm{SCl}$ patients, appropriate imaging studies should be obtained without delay.

Keywords: Acute abdomen; Emergency surgery; Spinal cord injury

\section{Introduction}

Spinal Cord Injury (SCI) is a catastrophic event with 15-40 new cases per million people per year [1]. The timely diagnosis of an acute abdomen in SCI patients represents a significant challenge due to the alterations in their abdominal somatic and visceral sensation. As a result, approximately $33 \%$ of SCI patients experience a significant delay (1-4 days) in the diagnosis and management of their abdominal emergencies [2-4], leading to increased mortality rates of $4-17 \%$ and morbidity rates of 30-50\% [3-9].

In order to facilitate early diagnosis and management of acute abdominal diseases in patients with spinal cord injury, it is crucial that surgeons become familiar with the neurological consequences of spinal cord injury and maintain a high index of suspicion for acute abdominal pathologies in this vulnerable population. The purpose of this paper is to provide a contemporary review of the diagnosis and management of abdominal emergencies in spinal cord injury patients.

\section{Dysfunction of Abdominal Innervation in SCI}

Spinal cord injury interrupts connections between central and peripheral nervous system, resulting in somatic motor and sensory deficits, as well as autonomic dysfunctions. The symptoms vary according to the level and severity of the injury. When evaluating SCI patients, surgeons should consider the type of neurologic deficit and the SCI level, because this influences the presenting signs and symptoms of acute abdomen.

\section{Somatic abdominal sensation}

Somatic abdominal sensation (T7-L1) is lost with lesions above T7 [3,5]. However, chronic SCI patients may retain some sensation over their anterior abdominal wall, suggesting that some innervation pathways may remain intact [5]. According to Neumayer LA, et al. [5], a potential explanation is that a large proportion of SCI is the result of blunt trauma in which the cord is damaged but not transected, resulting in partial preservation of innervation.

\section{Abdominal visceral sensation}

While somatic sensation is compromised in SCI patients, visceral sensation may often be present. Although the precise mechanism remains undetermined, one reason could be that visceral sensory nerves travel along the autonomic ganglia and enter the cord above the level of injury [10]. Visceral sensory fibers could also travel with the vagus nerve, thus bypassing the cord lesion [5]. In addition, some visceral afferent pathways may remain intact through the level of injury [5]. 


\section{Enteric nervous system}

Small and large bowel activity is mostly autonomous, controlled by the Enteric Nervous System (ENS), in addition to signals from the spinal cord and the brain [11]. ENS is an intrinsic nervous system of the intestine which includes Auerbach's plexus between the longitudinal and the circular muscle layers, and Meissner's plexus in the submucosa [12]. The ENS is modulated by central input from parasympathetic and sympathetic nerves (Figure 1). The vagus nerve provides parasympathetic regulation from the esophagus all the way to the splenic flexure of colon. Parasympathetic fibers originating from the S2-S4 spinal levels join the descending colon and rectum via the pelvic nerve.

The splanchnic nerves from the T5-T12 spinal levels and the hypogastric nerve from the T12-L2 levels provide sympathetic preganglionic innervation to postganglionic neurons controlling the colon. The external anal sphincter muscle is innervated bysomatic pudendal nerve from the S2-S4 levels [13].

The ENS remains functionally intact after spinal cord injuries [13]. However, spinal cord injuries cause changes in the neuronal control of the bowel through local synaptic remodeling [14,15], leading to colonic dysfunction known as "neurogenic bowel".

Spinal cord injuries above the conus medullar is result in an upper motor neuron syndrome characterized by increased colonic and anal sphincter tone. The striated external anal sphincter muscle, normally under voluntary control, remains tight as a result of losing descending inhibitory regulation [16]. With time this colonic outflow obstruction leads to constipation and stool retention causing colonic elongation and abdominal distension.

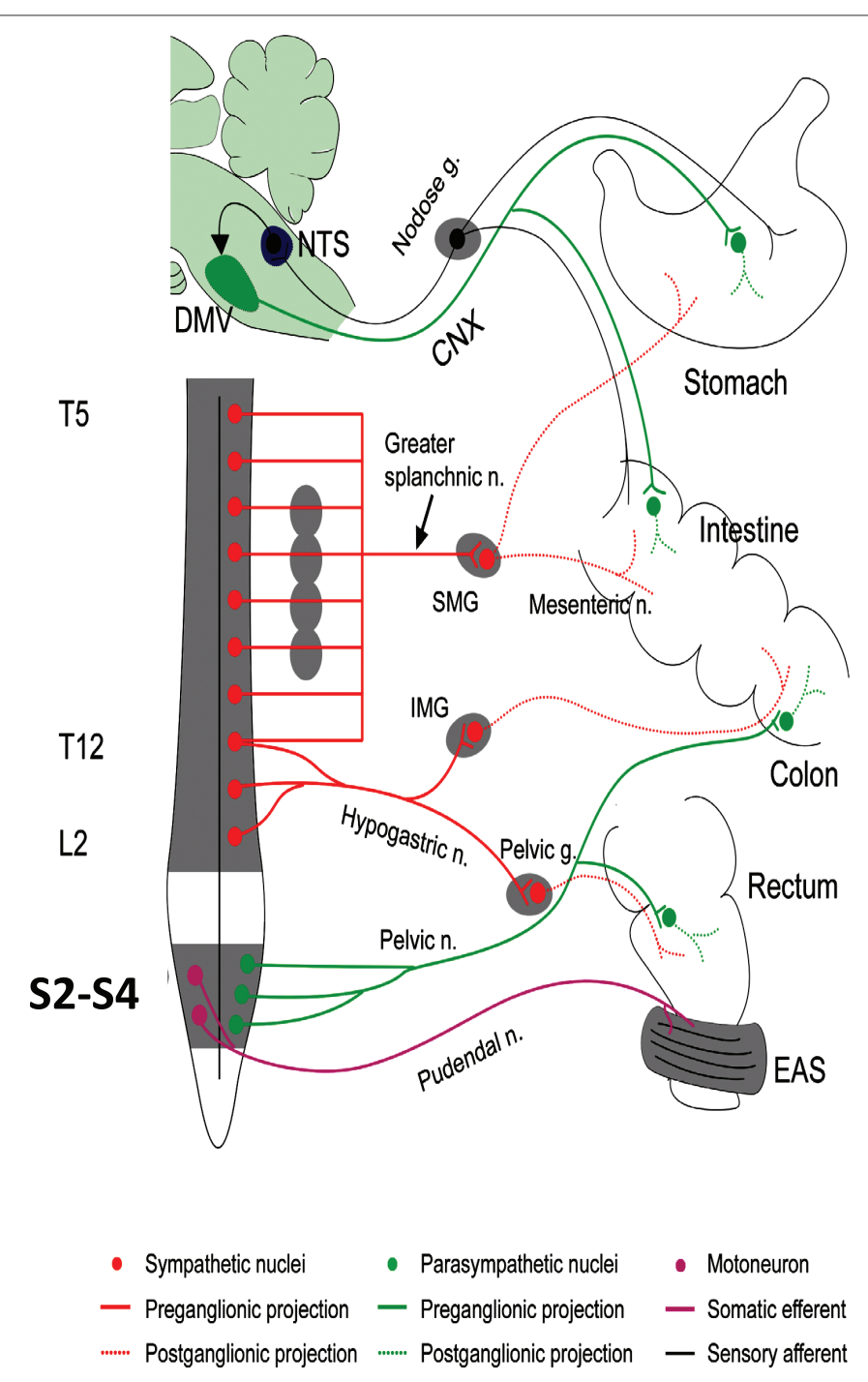

Figure 1: Schematic representation of neuronal control of the gastrointestinal tract (adapted from Hou S, et al. [11]).

The Vagus Nerve (CNX) provides parasympathetic regulation from the esophagus to the splenic flexure of colon. Ascending afferent fibers terminate in the Nucleus Tractus Solitarii (NTS) where the signals are integrated and subsequently relayed to parasympathetic neurons in the Dorsal Motor Nucleus of Vagus (DMV). Parasympathetic fibers originating from the S2-S4 spinal levels join the descending colon and rectum via the pelvic nerve. The mesenteric nerves from the T5-T12 spinal levels and the hypogastric nerve from theT12-L2 levels provide sympathetic preganglionic innervation to postganglionic neurons controlling the colon. The external anal sphincter muscle is innervated by somatic pudendal nerve from the S2-S4 levels. (CNX: Cranial Nerve X; SMG: Superior Mesenteric Ganglion; IMG: Inferior Mesenteric Ganglion; G: Ganglion; N: Nerve). 
In contrast, the lower motor neurogenic bowel results from injuries below the conus medullaris (such as cauda equina or pelvic nerve injuries), which damage motor and parasympathetic pathways to the colon and rectum [13]. These lesions are characterized by a severe rectosigmoid dysfunction with a relaxed colon and slow stool propulsion, leading to constipation [13]. In addition, the external anal sphincter becomes denervated an atonic, increasing the risk of incontinence. Subsequently, the levator animuscles lose tone and the sigmoid and rectum descend into the pelvic floor, contributing to fecal incontinence as well development of hemorrhoids [17].

\section{Autonomic dysreflexia}

Autonomic Dysreflexia (AD) is a syndrome characterized by a profound disordered autonomic response to visceral or cutaneous stimuli below the level of spinal cord lesion [13]. Clinical manifestations include a sudden increase in blood pressure of at least $20 \mathrm{mmHg}$ from baseline, baroreflex-mediated bradycardia, headache, flushing, sweating, chills, nasal congestion, piloerection, and pallor.

Severe hypertension can lead to raised intracranial pressure resulting in seizures, intracranial hemorrhage, myocardial ischemia, arrhythmias, pulmonary edema, and even death [18].

Development of $\mathrm{AD}$ is influenced by the duration and the level of the spinal injury. Although $\mathrm{AD}$ may develop any time following spinal cord injuries, it usually occurs in chronic SCI patients. The incidence of $\mathrm{AD}$ is between $50 \%$ and $70 \%$ in patients with lesions above T6 [19] and increases in frequency with higher level lesions and complete lesions [20]. The higher the level of spinal cord injury, the more severe the autonomic dysreflexia symptoms $[13,18]$.

The pathophysiology of $\mathrm{AD}$ is thought to be a result of a disorganized sympathetic response to stimuli below the level of the lesion (Figure 1). The loss of supraspinal inhibitory modulation of spinal sympathetic neurons facilitates a massive discharge of sympathetic preganglionic neurons below the level of the lesion, which can give rise to a profound sympathetic response up to the level of injury, with alterations in vasomotor, pilomotor, and sudomotor activities [13].

When spinal cord injuries are above the T6 level, the splanchnic innervation is activated, causing splanchnic vasoconstriction and resulting in more severe symptoms such as hypertension and compensatory bradycardia.

In patients with SCI below the T6 spinal level, supraspinal sympathetic innervation of the splanchnic vasculature is usually preserved; as a result, autonomic dysreflexia rarely occurs [18].

$\mathrm{AD}$ is typically triggered by noxious pelvic visceral stimulation, with the most common (approximately 80\%) cause being bladder distension [19]. Other triggers include acute abdominal pathology, bowel distension, urinary tract infections, skeletal fractures, pressure ulcers, activation of pain fibers, sexual activity, uterine contractions, and even gentle skin stroking [19,21-23].

\section{Diagnostic Features of Acute Abdomen in SCI Patients}

\section{Symptoms}

In neurologically intact patients, abdominal pain is the most common presenting symptom of an acute abdomen. Because SCI patients have altered symptoms of acute abdominal processes, timely diagnosis of acute abdomen becomes more challenging. As such, surgeons should be aware of signs and symptoms in the neurologically impaired patient that can facilitate earlier diagnosis of an acute abdomen.
Early symptoms of an intra-abdominal pathology in SCI patients are often subtle and non-specific, such as deviations from the SCI patient's normal lifestyle, feeling that "something is wrong", anorexia, and nausea $[6,24]$.

The early pain of intra-abdominal pathologies in SCI patients is due to inflammation of visceral peritoneum and is usually described as dull and oppressive $[7,25]$. As the inflammation progresses and involves the parietal peritoneum, SCI patients experience somatic pain that is described as sharp and severe. The reason for this difference between visceral and somatic pain is the slow rate of transmission of visceral sensation over unmyelinated class $\mathrm{C}$ fibers at 2 meters/sec, compared to a rate of 100 meters/sec for the sensation conducted via the large myelinated somatic sensory fibers [25].

Usually the pain of an acute abdomen in patients with low (below T6) spinal cord injuries is better localized and similar to normal patients $[3,5,7]$, whereas pain is more likely to be absent or vague in high (above T6) cord lesions [3,5]. However, patients with incomplete high cord lesions may experience normal pain sensation [7].

Another important symptom is referred pain to the shoulder $[3,7,9,26]$, which is more common in high (above T6) spinal cord injuries [5,7].

More than $80 \%$ of the SCI patients experience some degree of muscle spasticity, resulting in involuntary and sustained contractions of muscles [27]. Spasticity is more prevalent in high cord injuries due to increased reflex activity. If spasticity or muscle spasms suddenly become worse than usual or the pattern of the spasticity changes, it may be a warning sign of an acute process such as a urinary tract infection, constipation, pressure wounds, fractures, or an acute abdomen. In some cases, increased spasticity in the abdominal or lower extremity musculature may be the only clue to significant intraabdominal pathology, especially when in association with abdominal pain $[6,7,9,24]$.

\section{Signs}

Fever occurs in $32 \%$ to $81 \%$ of SCI patients with acute abdomen $[3,5,7,9]$, and are usually due to urinary tract infections $[6,7,28,29)]$.

Abdominal distension is encountered in $27 \%$ to $77 \%$ of patients $[3,5,8]$. However, abdominal distension is commonly encountered in SCI patient in the absence of an acute abdomen and is due to chronic constipation and gastroparesis. As such, abdominal distension has a diagnostic value when there is an acute increase in girth of the abdomen $[7,8]$.

Autonomic dysreflexia (21-92\%), tenderness with palpation (50\%), palpable abdominal mass (23\%), rigidity (14-21\%), and hypoactive bowel sounds (18\%) are some other findings of acute abdomen in SCI patients $[3,5,7,8,30]$. Surgeons should be aware that abdominal rigidity may not be easily recognized in SCI patients because of chronic spasticity or flaccidity of the abdominal muscles [6]. However, increased resistance can be felt over the affected region of the abdomen or can present as a change in the characteristic of abdominal wall spasticity from tonic to clonic $[10,31]$.

\section{Laboratory findings}

Laboratory tests used to differentiate diagnosis in SCI patients with acute abdomen include a complete blood count, amylase, lipase, liver enzymes, basic chemistry, and urinalysis. However, surgeons should remain aware that laboratory results are not reliable in the diagnosis of acute abdomen in the patient with SCI. Leukocytosis is not specific to acute abdomen as most of these patients are prone 
to concurrent conditions such as respiratory infections, urinary tract infections, and pressure wounds which can induce a rise in the leukocyte count. In addition, leukocytosis is reported only in 33-55\% of acute abdomens [3,5]. Furthermore, while a white cell left shift is found in most neurologically intact patients with an acute abdomen, is has been reported only in 36\% of SCI patients [5]. Additionally, a positive urinalysis in this population does not equate an infection, but rather colonization of the urine secondary to chronic indwelling catheterizations required by many SCI patients with neurogenic bladder.

\section{Radiologic findings}

Given the significant challenge of correctly diagnosing an acute abdomen in SCI patients based on clinical features alone, surgeons should have a low threshold for obtaining appropriate radiographic studies to facilitate early diagnosis. In 77\% of SCI patients radiographic studies are required to correctly diagnosing acute intra-abdominal pathology [5].

Although abdominal X-ray is often the first imaging test obtained [32], the most useful diagnostic imaging tests are ultrasound and computed tomography.

\section{Acute Abdominal Diseases in SCI Patients}

\section{Acute cholecystitis}

Cholecystitis occurs in $16.7-36 \%$ of SCI patients with an acute abdomen $[5,6,30,33]$ and is attributed to a propensity for the development of gallstones in this population. Thus, the prevalence of gallstones in SCI patients is $17-49 \%$ [34-36], compared to $7-15 \%$ in neurologically intact patients $[30,35,36]$. The mechanism underlying the association between gallstones and SCI remains unclear; however, there is evidence of gallbladder dysfunction in SCI patients. While gallbladder contraction remains normal in SCI patients $[37,38]$, gallbladder relaxation is often impaired in injuries above the T10 level [37-39], resulting in lower resting volumes. Ultrasonographic studies have found lower resting volume in SCI patients $(21 \mathrm{ml})$ compared with healthy subjects $(28 \mathrm{ml})$, suggesting that lower resting volume may increase the bile lithogenesis [37], leading to cholelithiasis.

The observed higher incidence of cholecystitis in SCI patients is responsible for a higher annual rate of cholecystectomy (6.3\%) than in the general population (3.7-5.1\%) [34]. Furthermore, given the diagnostic delay of cholecystitis, SCI patients have a higher annual incidence of biliary complications (2.2\%) than the general population $(0.2 \%$ to $1.0 \%)$ [34]. Although mortality rates are comparable to the general population, SCI patients have a higher complication rate and a higher conversion from laparoscopic to open procedures [34].

\section{Acute appendicitis}

The incidence of acute appendicitis in SCI patients is 4.5-23.8\% [57]. Although a palpable right lower quadrant mass can be found in $23 \%$ of patients, only $35 \%$ of cases are diagnosed correctly by clinical features [8]. Therefore, imaging studies are very important for a timely diagnosis, with CT scan being the most helpful investigation. In a study of 26 SCI patients with appendicitis, delays in diagnosis resulted in perforated appendicitis in $92 \%$ of cases, requiring more extensive surgeries. Thus, $88 \%$ of patients underwent a standard appendectomy, with $12 \%$ requiring a concomitant cecal resection [8]. This resulted in a high mortality $(4 \%)$, increased postoperative complications $(23 \%)$ and prolonged length-of-stay (mean of 16 days) [8].

\section{Intestinal volvulus}

Chronic constipation and stool retention result in a long redundant colon [40], and predispose SCI patients to colonic volvulus, which has been reported in $4.2-12.5 \%$ of cases $[5,6,9]$.

Furthermore, fecal retention in the ascending colon leads to distal obstruction and dilation of small bowel in $39 \%$ of SCI patients [41], resulting in small bowel volvulus in $16.7-25 \%$ of cases $[6,9]$.

\section{Perforated peptic ulcers}

The incidence of peptic ulcers in SCI patients remains higher than in the general population [42-46]. Most peptic ulcers occur in the first month after the acute cord injury, with an incidence of $1-24 \%$ $[42,45,47]$, whereas the long-term incidence is $1-5 \%$ [43].

Peptic ulcers are more common in SCI patients with injuries above theT4 level, because sympathetic denervation of the thoraco-lumbar outflow tract creates an imbalance between the parasympathetic and sympathetic innervation of the stomach and duodenum. This result in increased gastric acid secretion, reduced perfusion of the gastric mucosa, decreased mucus production, impaired prostaglandin synthesis, decreased gastricantral contractions, bile reflux secondary to pyloric incompetence, delayed gastric emptying, and atrophic gastritis $[42,44,45,47]$. Because of delays in clinical presentations, peptic ulcers in SCI patients are more severe and have more complications [48]. Thus, perforated gastro duodenal ulcers occur in $12.5-25 \%$ of cases $[5,6,9]$. In addition to a high index of clinical suspicion, imaging studies are particularly important for early diagnosis of perforated peptic ulcers given the paucity of clinical features.

Therefore, surgeons should remain familiar with the radiographic characteristics of intra-peritoneal air secondary to perforated viscus. An upright chest $\mathrm{x}$-ray can detect as little as $1 \mathrm{ml}$ of intraperitoneal air under the diaphragm, and is the most sensitive plain radiographic study for the detection of free intraperitoneal air. However, supine abdominal X-ray is usually obtained as the first line investigation by physicians caring for SCI patients. Therefore, it is important to become familiar with the correct interpretation of supine abdominal X-rays in order to avoid missing the critical diagnosis of pneumoperitoneum and to expedite the management of perforated peptic ulcers.

An important feature of pneumoperitoneum on a supine abdominal $\mathrm{X}$-ray is the Rigler's sign, also known as the double-wall sign. Rigler's sign is present when gas outlines both serosal and luminal surfaces of the bowel wall $[49,50]$ (Figure 2). Unfortunately, large amounts of pneumoperitoneum $(>1000 \mathrm{~mL}$ ) are usually required to demonstrate this sign [51]. The positive predictive value of Rigler's sign is $74 \%$ [52], highlighting its important diagnostic value in SCI patients with perforated viscus.

Computer tomography would also demonstrate signs of pneumoperitoneum and has the advantage of further delineating the source of the perforation.

\section{Other conditions}

The differential diagnosis of acute abdomen in SCI patients is quite broad and must span to include various other conditions such as intestinal obstruction $[5,7,9]$, perforation of large and small bowel $[6,53]$, bladder perforation caused by chronic indwelling urinary catheters [5-7] fulminant pseudomembranous colitis [54,55]; toxic megacolon [56]; complications of inflammatory bowel disease [57,58], upper gastrointestinal bleeding [6]; complicated acute pancreatitis [59]; retroperitoneal hemorrhage [6], pelvic hematoma [30]; feeding tube dislodgement [60]; and superior mesenteric artery syndrome [61]. 


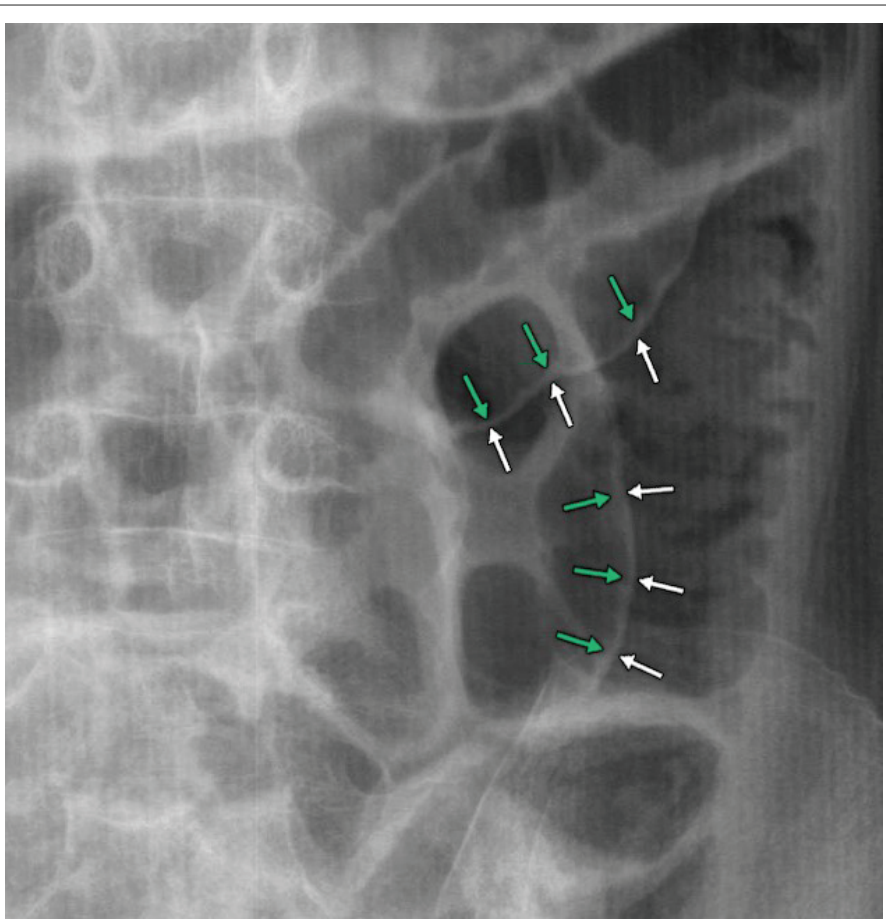

Figure 2: Supine abdominal X-ray showing the Rigler's (double-wall) sign (adapted from Kabbani AA, et al. [50])

Free intra-peritoneal air outlining both serosal (white arrows) and luminal (green arrows) surfaces of the bowel wall.

\section{Conclusions}

Early diagnosis of acute abdomen in SCI patients is the most important factor for improving outcomes. However, the clinical recognition of abdominal emergencies in SCI patients remains challenging due to alterations in their abdominal somatic and visceral sensation, as well as coexisting chronic abdominal conditions prevalent in this group of patients. Therefore, in addition to understanding the neurological consequences of spinal cord injury and maintaining a high clinical suspicion for abdominal emergencies in SCI patients, appropriate imaging studies should be obtained without delay. Furthermore, it is crucial for surgeons to maintain competency in the prompt interpretation of radiographic studies in order to expedite the management of acute emergencies in SCI patients.

\section{References}

1. Sekhon LH, Fehlings MG (2001) Epidemiology, demographics, and pathophysiology of acute spinal cord injury. Spine (Phila Pa 1976) 26: S2-S12.

2. Zaremski JL, Jayawardena V, Lew HL (2010) An occult presentation of appendicitis in a patient with tetraplegia. Am J Phys Med Rehabil 89: 156-159.

3. Miller BJ, Geraghty TJ, Wong CH, Hall DF, Cohen JR (2001) Outcome of the acute abdomen in patients with previous spinal cord injury. ANZ J Surg 71: 407-411.

4. Sheridan R (1992) Diagnosis of the acute abdomen in the neurologically stable spinal cord-injured patient. A case study. J Clin Gastroenterol 15: 325-328.

5. Neumayer LA, Bull DA, Mohr JD, Putnam CW (1990) The acutely affected abdomen in paraplegic spinal cord injury patients. Ann Surg 212: 561-566.
6. Charney KJ, Juler GL, Comarr AE (1975) General surgery problems in patients with spinal cord injuries. Arch Surg 110: 1083-1088.

7. Juler GL, Eltorai IM (1985) The acute abdomen in spinal cord injury patients. Paraplegia 23: 118-123.

8. Strauther GR, Longo WE, Virgo KS, Johnson FE (1999) Appendicitis in patients with previous spinal cord injury. Am J Surg 178: 403-405.

9. Bar-On Z, Ohry A (1995) The acute abdomen in spinal cord injury individuals. Paraplegia 33: 704-706.

10. Hoen T, Cooper IS (1948) Acute abdominal emergencies in paraplegics. A J Surgery 75: 19-24.

11. Hou S, Rabchevsky AG (2014) Autonomic consequences of spinal cord injury. Compr Physiol 4: 1419-1453.

12. Goyal RK, Hirano I (1996) The enteric nervous system. N Engl J Med 334: 1106-1115.

13. Sarna SK (1991) Physiology and pathophysiology of colonic motor activity. Dig Dis Sci 36: 827-862.

14. Devroede G, Arhan P, Duguay C, Tetreault L, Akoury H, et al. (1979) Traumatic constipation. Gastroenterology 77: 1258-1267.

15. Devroede G, Lamarche J (1974) Functional importance of extrinsic parasympathetic innervation to the distal colon and rectum in man. Gastroenterology 66: 273-280.

16. Shafik A (1995) Electrorectogram study of the neuropathic rectum. Paraplegia 33: 346-349.

17. Bartolo DC, Read NW, Jarratt JA, Read MG, Donnelly TC, et al. (1983) Differences in anal sphincter function and clinical presentation in patients with pelvic floor descent. Gastroenterology 85: 68-75.

18. Petsas A, Drake J (2015) Perioperative management for patients with a chronic spinal cord injury. BJA Education 15: 123-130. 
19. Shergill IS, Arya M, Hamid R, Khastgir J, Patel HR, et al. (2004) The importance of autonomic dysreflexia to the urologist. BJU Int 93: 923-926.

20. Tomassoni PJ, Campagnolo DI (2003) Autonomic dysreflexia: One more way EMS can positively affect patient survival. JEMS 28: 46-51.

21. Karlsson AK (1999) Autonomic dysreflexia. Spinal Cord 37: 383-391.

22. Krassioukov AV, Johns DG, Schramm LP (2002) Sensitivity of sympathetically correlated spinal interneurons, renal sympathetic nerve activity, and arterial pressure to somatic and visceral stimuli after chronic spinal injury. J Neurotrauma 19: 1521-1529.

23. Lindan R, Joiner E, Freehafer AA, Hazel C (1980) Incidence and clinical features of autonomic dysreflexia in patients with spinal cord injury. Paraplegia 18: 285-292.

24. Cho KT, Lee SC, Lee HJ, Kang HW (2013) Acute abdomen in a cervical spinal cord injury patient: a case report and review of the literature. Hong Kong Journal of Emergency Medicine 20: 178-181.

25. Ingberg H, Prust $F(1968)$ The diagnosis of abdominal emergencies in patients with spinal cord lesions. Arch Phys Medicine Rehab 49: 343-348.

26. Mowrey K (2007) The challenge of assessing and diagnosing acute abdomen in tetraplegics: a case study. J Neurosci Nurs 39: 5-8.

27. Levi R, Hultling C, Seiger A (1995) The Stockholm spinal cord injury study: Associations between clinical patient characteristics and post-acute medical problems. Paraplegia 33: 585-594.

28. Sugarman B, Brown D, Musher D (1982) Fever and infection in spinal cord injury patients. JAMA 248: 66-70.

29. Miller LS, Staas We, Herbison GJ (1975) Abdominal problems in patients with spinal cord lesions. Arch Phys Med Rehabil 56: 405-408.

30. Sarıfakıoğlu B, Afşar SI, Yalbuzdağ ŞA, Ustaömer K, Ayaş Ş (2014) Acute abdominal emergencies and spinal cord injury; our experiences: a retrospective clinical study. Spinal Cord 52: 697-700.

31. Greenfield J (1949) Abdominal operations on patients with chronic paraplegia. Arch Surg 59: 1077-1087.

32. Rajan RK, Nemchausky BA (2001) Gastrointestinal emergencies in patients with spinal cord injury. In: Eltorai MI, Schmitt JK (eds) Emergencies in chronic spinal cord injury patients. Eastern Paralyzed Veterans Association.

33. Xia CS, Han YQ, Yang XY, Hong GX (2004) Spinal cord injury and cholelithiasis. Hepatobiliary Pancreat Dis Int 3: 595-598.

34. Moonka R, Stiens SA, Resnick WJ, McDonald JM, Eubank WB, et al. (1999) The prevalence and natural history of gallstones in spinal cord injured patients. J Am Coll Surg 189: 274-281.

35. Tola VB, Chamberlain S, Kostyk SK, Soybel DI (2000) Symptomatic gallstones in patients with spinal cord injury. J Gastrointest Surg 4: 642-647.

36. Everston GT (1991) Gallbladder function in gallstone disease. Gastroenterol Clin North Am 20: 84-110.

37. Nino-Murcia M, Burton D, Chang P, Stone J, Perkash I (1990) Gallbladder contractility in patients with spinal cord injuries: a sonographic investigation. AJR Am J Roentgenol 154: 521-524.

38. Tandon RK, Jain RK, Garg PK (1997) Increased incidence of biliary sludge and normal gallbladder contractility in patients with high spinal cord injury. Gut 41: 682-687.

39. Ketover SR, Ansel HJ, Goldish G, Roche B, Gebhard RL (1996) Gallstones in chronic spinal cord injury: is impaired gallbladder emptying a risk factor? Arch Phys Med Rehabil 77: 1136-1138.
40. Fenton-Lee D, Yeo BW, Jones RF, Engel S (1993) Colonic volvulus in the spinal cord injured patient. Paraplegia 31: 393-397.

41. Harari D, Minaker KL (2000) Megacolon in patients with chronic spinal cord injury. Spinal Cord 38: 331-339.

42. Kewalramani LS (1979) Neurogenic gastroduodenal ulceration and bleeding associated with spinal cord injuries. J Trauma 19: 259-265.

43. Tanaka M, Uchiyama M, Kitano M (1979) Gastroduodenal disease in chronic spinal cord injuries. An endoscopic study. Arch Surg 114: 185-187.

44. Leramo OB, Tator $\mathrm{CH}$, Hudson AR (1982) Massive gastroduodenal hemorrhage and perforation in acute spinal cord injury. Surg Neurol 17: 186-190.

45. Soderstrom CA, Ducker TB (1985) Increased susceptibility of patients with cervical cord lesions to peptic gastrointestinal complications. J Trauma 25: 1030-1038.

46. Albert TJ, Levine MJ, Balderston RA, Cotler JM (1991) Gastrointestinal complications in spinal cord injury. Spine 16: S522-S525.

47. Lu WY, Rhoney DH, Boling WB, Johnson JD, Smith TC (1997) A review of stress ulcer prophylaxis in the neurosurgical intensive care unit. Neurosurgery 41: 425-426.

48. Park WG, Rouse RV, KahngLS, Bastidas JA, Meinke L, et al. (2007) Giant gastric ulcers in chronic spinal cord injury patients. Digestive Endoscopy 19: 36-39.

49. Rigler LG (1941) Spontaneous pneumoperitoneum: a roentgenologic sign found in the supine position. Radiology 37: 604-607.

50. Kabbani AA, Jones J (2020) Rigler'signs (bowel). Radiopaedia.

51. Schultz EH Jr (1958) An aid to the diagnosis of pneumoperitoneum from supine abdominal films. Radiology 70: 728-731.

52. Levine MS, Scheiner JD, Rubesin SE, Laufer I, Herlinger H (1991) Diagnosis of pneumoperitoneum on supine abdominal radiographs. AJR Am J Roentgenol 156: 731-735.

53. Matsuo A, Tokuyama $Y$, Hosono $Y$, Hiraoka T, Furuta $T$ (2004) Ileal perforation in a patient with high spinal cord injury: report of a case. Surg Today 34: 65-67.

54. Johnson DK, Balmaseda MT (1985) Pseudomembranous colitis in spinal cord injury. Arch Phys Med Rehabil 66: 394-396.

55. Bahadursingh AN, Vagefi PA, Longo WE (2004) Fulminant Clostridium difficile colitis in a patient with spinal cord injury: case report. $J$ Spinal Cord Med 27: 266-268.

56. Culig MH, Kelly EG, Layton TR (1984) Toxic megacolon following spinal cord injury. Pa Med 87: 51-52.

57. Yung JC, Groah SL (2001) Crohn's disease in a patient with acute spinal cord injury: a case report of diagnostic challenges in the rehabilitation setting. Arch Phys Med Rehabil 82: 1274-1278.

58. Han SJ, Kim CM, Lee JE, Lee TH (2009) Colonoscopic lesions in patients with spinal cord injury. J Spinal Cord Med 32: 404-407.

59. Pirolla EH, de Barros Filho TE, Godoy-Santos AL, Fregni F (2014) Association of acute pancreatitis or high level of serum pancreatic enzymes in patients with acute spinal cord injury: a prospective study. Spinal Cord 52: 817-820.

60. Hess MJ, Foo DK (2010) Percutaneous enterogastric tube dislodgement in tetraplegics. Spinal Cord 48: 83-84.

61. Kim SJ, Yoon JS, Park BK, Won SJ (2010) Superior mesenteric artery syndrome in a tetraplegic patient, 11 years after a spinal cord injury: a case report. Spinal Cord 48: 838-839. 